\title{
MAXIMAL SEMICHARACTERS
}

\section{ROBERT KAUFMAN ${ }^{1}$}

In this note the following notations are used.

$G$ denotes a commutative semigroup with identity 1 and $H$ a subsemigroup containing 1 .

$N$ is a real-valued function on $G$ such that (i) $N \geqq 0$ (ii) $N(1)=1$ (iii) $N(x y) \leqq N(x) N(y)$ for elements $x, y$ in $G$.

A real homomorphism $\phi$ is a function on $G$ (or $H$ ) satisfying (i), (ii) and $\phi(x y) \equiv \phi(x) \phi(y)$.

Our interest is in real homomorphisms subject to the inequality $N \geqq \phi \geqq 0$, and especially in homomorphisms of this type which are maximal in that $N \geqq \sigma \geqq \phi \geqq 0$ implies $\phi=\sigma$.

TheOREM 1. Suppose that $\phi$ is a real homomorphism of $H$ and

(iv) $a h_{1}=b h_{2}\left(a, b \in G, h_{i} \in H\right)$ implies $N(a) \phi\left(h_{1}\right) \geqq \phi\left(h_{2}\right)$.

Then $\phi$ can be extended to a real homomorphism $\sigma$ for which $N \geqq \sigma \geqq 1$.

Proof. In the family $\left\{N^{\prime}\right\}$ of functions $N^{\prime} \leqq N$ which fulfill requirements (i)-(iv) there is evidently a minimal element and we can assume that the given function $N$ is itself that minimal function. Under these circumstances it is to be proved that $\sigma \equiv N$ is the real homomorphism required. The method of proof is the construction of a function $M$ which fulfills (i)-(iv) and is finally identified with $N$.

We consider the equations (vi) $a h_{1}=x^{n} b h_{2}\left(a, b, x \in G, h_{i} \in H, n \geqq 1\right.$ ). For each $x$, let $M(x)=\inf \left\{\phi\left(h_{1}\right) N(a) / \phi\left(h_{2}\right)\right\}^{1 / n}$, the infimum taken over all solutions of (vi). The verification that $M$ satisfies (ii)-(iv) follows.

Writing $a=b=h_{1}=h_{2}=x=1$ yields $M(1) \leqq 1$ and if $a h_{1}=b h_{2}$, $N(a) \phi\left(h_{1}\right) \geqq \phi\left(h_{2}\right)$ by (iv). To prove (iii) for $M$, suppose in addition to (vi) that $c h_{3}=d y^{m} h_{4}$. Then

$$
a^{m} c^{n} \cdot h_{1}^{m} h_{3}^{n}=b^{m} d^{n} \cdot(x y)^{m n} h_{2}^{m} h_{4}^{n}
$$

whence

$$
M(x y) \leqq\left\{\frac{\phi\left(h_{1}\right)^{m} \phi\left(h_{3}\right)^{n} N\left(a^{m} c^{n}\right)}{\phi\left(h_{2}\right)^{m} \phi\left(h_{4}\right)^{n}}\right\}^{1 / m n}
$$

which is less than the product of the corresponding ratios for $M(x)$ and $M(y)$.

Received by the editors September 3, 1965.

1 Supported in part by the National Science Foundation. 
For obvious reasons (iv) is attacked indirectly; we show first that $M \equiv \phi$ in $H$. To see that $M \geqq \phi$ in $H$ suppose that $a h_{1}=h^{n} b h_{2}$. Applying (iv) to the function $N, N(a) \phi\left(h_{1}\right) \geqq \phi(h)^{n} \phi\left(h_{2}\right)$ so $M(h) \geqq \phi(h)$. In the reverse sense $1 \cdot h=1 \cdot h \cdot 1$ yields immediately $M(h) \leqq \phi(h)$. We observe also that $M(x y) \geqq M(x)$, for if $a h_{1}=b(x y)^{n} h_{2}$ we have $a h_{1}$ $=b y^{n} \cdot x^{n} \cdot h_{2}$.

The proof that $M$ satisfies (iv) is now easy: $M(a) \phi\left(h_{1}\right)=M(a) M\left(h_{1}\right)$ $\geqq M\left(b h_{2}\right) \geqq M\left(h_{2}\right)$. Trivially $M \leqq N$ so $M=N$.

If $x \in G$ and $m \geqq 1, M\left(x^{m} h\right)=M(x)^{m} M(h)$ or equivalently $M(x)^{m} M(h) \leqq M\left(x^{m} h\right)$. To see this suppose $a h_{1}=\left(x^{m} h\right)^{n} b h_{2}$ in (iv); then since $a h_{1}=x^{m n} \cdot b \cdot h^{n} h_{2} M(x) \leqq\left\{N(a) \phi\left(h_{1}\right) / \phi\left(h_{2}\right) \phi(h)^{n}\right\}^{1 / m n}$ which yields the asserted inequality.

Thus $M$ is multiplicative in the semigroup generated by $H \cup\{x\}$; since $M(=N)$ was minimal, repetition of the same argument shows that $M$ is multiplicative in the semigroup generated by $H \cup\{x, y\} \cdots$. Since $M(x \cdot 1) \geqq M(1)=1$ is known, the proof of Theorem 1 is complete.

The necessity of (iv), one observes, is obvious.

Theorem 2. Suppose $a \in G, r>1$ and $N\left(a^{n} x\right) \geqq r^{n}$ for all $x \in G, n \geqq 1$. Then there is a real homomorphism $\phi$ of $G$ such that $\phi(a)=r$ and $N \geqq \phi \geqq 1$.

Proof. $H$ is taken here to be the semigroup of 1 and the powers of $a$. Since $N\left(a^{n}\right) \geqq r^{n}$ for each $n$ it is clear $a^{m} \neq a^{n}$ when $m>n \geqq 0$, for if equality is obtained $H$ would be finite. Thus we can define $\phi\left(a^{n}\right)=r^{n}$ for $n \geqq 0$ and turn to the hypotheses of Theorem 1 .

I. $x a^{n}=y a^{m}$ and $m=n+p>n \geqq 0$. Rearranging, $x a^{n}=y a^{p} \cdot a^{n}$ and then for each integer $s \geqq 1, x^{s} a^{n}=\left(y a^{p}\right)^{s} \cdot a^{n}$, inductively: if the last equality holds,

$$
x^{s+1} a^{n}=\left(y a^{p}\right)^{s} \cdot x a^{n}=\left(y a^{p}\right)^{s+1} a^{n} .
$$

Then

$$
N(x)^{s} N(a)^{n} \geqq N\left(y^{s} a^{p s+n}\right) \geqq r^{p s+n} .
$$

Taking the $s$-root and letting $s \rightarrow \infty$ gives $N(x) \geqq r^{p}$ or

$$
N(x) r^{n} \geqq r^{p+n}=r^{m} .
$$

II. $x a^{n}=y a^{m}$ and $n \geqq m$. Inasmuch as $r^{n} \geqq r^{m}$ it is enough to check that $N \geqq 1$ in $G$. But

$$
N(x)^{s} N(a) \geqq N\left(x^{s} a\right) \geqq r>0
$$

so $N(x) \geqq 1$. 
Theorem 1 now gives the proof of Theorem 2 .

THEOREM 3. Let $\phi$ be a real homomorphism of $H$ such that $N \geqq \phi \geqq 0$ and $\phi$ is maximal among all homomorphisms with this property. Then $\phi$ can be extended to a homomorphism $\sigma$ of $G, N \geqq \sigma \geqq 0$.

Proof. $\phi$ is maximal also in the subset $S$ of $H$ in which $\phi>0$. For if $N \geqq \chi \geqq \phi \geqq 0$ in $S, \chi$ can be extended to $H$ by setting it equal to zero in $H-S$ so $\chi=\phi$ in $S$. If then $\phi$ can be extended from $S$ to a real homomorphism $\sigma$ of $G, N \geqq \sigma \geqq 0$, necessarily $\sigma \geqq \phi$ in $S \cup(H-S)$ so $\sigma=\phi$ in $H$. In brief we can suppose $\phi>0$ in all of $H$.

The hypothesis that $\phi>0$ be maximal can be restated: if $N / \phi \geqq \rho$ $\geqq 1$ for a homomorphism $\rho$ of $H$, then $\rho=1$. But $N / \phi$ satisfies the axioms (i)-(iii) and Theorem 2 is applicable for $N / \phi=N_{1}$.

The proof of Theorem 3 is now completed by the criterion of [1] for the existence of the required homomorphism $\sigma$. Namely: $x_{0} \in G$, $h_{0} \in H$ and $x_{0} h_{0} \in H$, implies that $N\left(x_{0}\right) \phi\left(h_{0}\right) \geqq \phi\left(x_{0} h_{0}\right)$.

The verification follows.

Given $r>1$ there exist by Theorem 2 an integer $n \geqq 1$ and an element $h \in H$ for which $N_{1}\left(h_{0}^{n} h\right) \leqq r^{n}$ or

$$
V\left(h_{0}^{n} h\right) \leqq r^{n} \phi\left(h_{0}\right)^{n} \phi(h) .
$$

But $x_{0} h_{0} \in H$ so that

$$
0<\phi\left(x_{0} h_{0}\right)^{n} \phi(h) \leqq N\left(x_{0}\right)^{n} N\left(h_{0}^{n} h\right) \leqq N\left(x_{0}\right)^{n} r^{n} \phi\left(h_{0}\right)^{n} \phi(h) .
$$

Finally $0<\phi\left(x_{0} h_{0}\right) \leqq r N\left(x_{0}\right) \phi\left(h_{0}\right)$ and Theorem 3 is proved.

REMARK. Hausdorff's principle may be used to prove the Theorem of [1], as Theorem 1 is proved here. This procedure is used in [2] to obtain a related result.

\section{REFERENCES}

1. Robert Kaufman, Extension of functions and inequalities in an Abelian semigroup, Proc. Amer. Math. Soc. 17 (1965), 83-85.

2. - Interpolation of additive functionals, Studia Math. (to appear).

UNIVERSITY OF ILLINOIS 